\title{
LINUS JUNG
}

Universidad de Granada

ljung@ugr.es

ORCID: 0000-0001-9356-8575

\author{
ANTONIO JAVIER CHICA NUÑEZ \\ Universidad Pablo de Olavide (Sevilla) \\ ajchinun@upo.es \\ ORCID: 0000-0001-9229-0014
}

\section{Intersemiotisches Übersetzen: Anmerkungen zur Audiodeskription aus einer übersetzungswissenschaftlichen Perspektive}

\author{
Intersemiotic translation: \\ Comments on audio description \\ from a Translation Studies perspective
}

\begin{abstract}
There is no doubt that Translation Studies have firmly established themselves as an independent scientific field. However, there are thematic overlaps in some areas concerning other disciplines. This work investigates the relations between interlinguistic translation and intersemiotic translation from the point of view of translation studies. First of all, a set of criteria for the identification of proper translation is offered and, in a second step, we examine how theoretical elements like communicative equivalence and communicative value can be transferred to a linguistic discourse by means of the intersemiotic translation modality known as audio description (AD).
\end{abstract}

KEYWORDS: intersemiotic translation, communicative equivalence, communicative value, audio description, visual object, visual context.

SCHLÜSSELWORTE: intersemiotisches Übersetzen, kommunikative Äquivalenz, kommunikativer Wert, Audiodeskription, visuelles Objekt, visueller Kontext. 


\section{EINLEITUNG ${ }^{1}$}

Als Übersetzen wird im Allgemeinen ein Kommunikationsprozess angesehen, der, vereinfacht gesagt, darauf beruht, dass ein Ausgangstext aus einer Sprache neu in einem Zieltext einer anderen Sprache entsteht. Nach der klassischen Defintion des Linguisten Roman Jakobson lassen sich dabei drei Grundarten des Übersetzens unterscheiden, nämlich die intralinguistische, die interlinguistische und intersemiotische Übersetzung (Jakobson 1959: 233), wobei die interlinguistische Übersetzung als das eigentliche Übersetzen angesehen wird, dem die Übersetzungswissenschaft ihre besondere Aufmerksamkeit bei ihren Untersuchungen und Studien schenkt.

Auch wenn das intersemiotische Übersetzen bei Jakobson noch auf das Übertragen eines sprachlichen Textes in ein nicht-sprachliches Kommunikationssystem beschränkt war, so hat gerade das intersemiotische Übersetzen in vielen Bereichen ein großes Forschungsinteresse gefunden, wie z.B. in der Vergleichenden Literaturwissenschaft, der Semiotik oder den intermedialen Studienansätzen. Generell bezeichnet man damit also Übertragungsprozesse, die sich zwischen unterschiedlichen Zeichensystemen vollziehen und eben nicht auf Sprachsysteme beschränkt sind. Als Beispiele für ein intersemiotisches Übersetzen lassen sich die Verfilmung (Aktulum 2017), die Vertonung (Kvam 2014), Bebilderung (da Silva 2017) und getanzte Versionen (Aguiar \& Queiroz 2013) von Literaturwerken anführen.

In diesem Beitrag möchten wir den intersemiotischen Übersetzungsprozess aus einer übersetzungswissenschaftlichen Perspektive näher beleuchten, um zu zeigen, dass dem intersemiotischen Übersetzen genau wie der Translation kognitive Bewusstseinsinhalte zu Grunde liegen und sich verschiedene Erkenntnisse der Translationswissenschaft übertragen lassen, die es dann erlauben, von einer Art des Übersetzens zu sprechen. Dabei soll die Audiodeskription als eine Art des intersemiotischen Übersetzens im Vordergrund stehen (vgl. Fernández, Martínez \& Chica 2015; Jiménez \& Martínez 2018).

\section{WAS IST UNTER ÜBERSETZEN ZU VERSTEHEN?}

Das Übersetzen besteht generell in einem Kommunikationsprozess, an dem zwei unterschiedliche Sprachen beteiligt sind, so dass aus einem Aus-

${ }^{1}$ Dieser Beitrag wurde innerhalb des Forschungsprojekts OPERA [Acceso al ocio y a la cultura. Plataforma de difusión y evaluación de recursos audiovisuales accesibles (FFI201565934-R)] erstellt, das vom spanischen Ministerio de Educación, Cultura y Deporte finanziert wird. 
gangstext (AT) in der Sprache $\mathrm{L}_{\mathrm{A}}$ ein Zieltext (ZT) in der Sprache $\mathrm{L}_{\mathrm{B}}$ entsteht. Es stellt sich aber nun die Frage, wie denn dieser Prozess vor sich geht. Reicht es aus zu sagen, es werden einfach die Wörter des AT im ZT wiedergegeben oder gibt es andere Kategorien, die es erlauben, den Übersetzungsvorgang $\mathrm{zu}$ beschreiben und wissenschaftlich zu erfassen? Dazu ist vor allem einmal in kürzester und einfachster Form abzuklären, erstens, wie Sprache an sich in der Kommunikation funktioniert, zweitens, worauf sich der Übersetzer bei seiner Tätigkeit eigentlich stützt, nämlich den kommunikativen Wert des AT, und drittens, in welchem Verhältnis der ZT zum AT stehen sollte, um überhaupt von einer Übersetzung sprechen zu können, bzw. welches Ziel bei einer Übersetzung im Allgemeinen verfolgt wird, nämlich die kommunikative Äquivalenz als die Beschreibung der Relation die zwischen AT und ZT bestehen muss, wenn man von Übersetzung im eigentlichen Sinn sprechen will.

\subsection{Wie funktioniert Sprache?}

Innerhalb der Übersetzungswissenschaft verstehen wir mit der bekannten Leipziger Schule (vgl. Wotjak 2006; Wotjak, Sinner, Jung \& Batista 2013) die Translation in ihren beiden Erscheinungsformen des Übersetzens und Dolmetschens als einen kommunikativen Akt, der den Gebrauch von zwei unterschiedlichen Sprachen im erwähnten Sinne von Jakobson (interlinguistische Übersetzung) voraussetzt. Für jede Art von sprachlicher Kommunikation, also auch der intra- und interlinguistischen, sind zwei grundlegende Fähigkeiten bei Verfasser und Adressaten entscheidend (Kade 1963: 85), ohne die eine annähernd erfolgreiche Verständigung fast unmöglich ist:

1. die Fähigkeit, eine kommunikative Intention mit sprachlichen Mitteln zu realisieren, d.h., eine Information mit Hilfe der Sprache mitteilbar zu machen;

2. die Fähigkeit, die in einer akustisch-phonetisch oder optisch-graphisch aufgenommenen Zeichenfolge enthaltene Information zu erkennen, d.h., die Zeichen zu dekodieren (Kade 1963: 85).

Dies bedeutet, dass gemäß diesen Kompetenzen zwei Funktionen bei der Sprachnutzung zum Tragen kommen. Jedes einzelne Wort einer Kommunikation und somit der Text als Ganzes hat eine repräsentative und eine kommunikative Funktion. Die repräsentative Funktion steht für einen Bewusstseinsinhalt, den sich der Autor als kognitiv-mentale Größe in Gedanken vorstellt und als seine Aussageintention mitzuteilen beabsichtigt. Gleichzeitig haben dann aber die Wörter eine kommunikative Funktion, wobei sie den vom Autor imaginierten Bewusstseinsinhalt und damit einen 
intendierten kommunikativen Effekt beim Adressaten auslösen können sollen. Kommunizieren heißt etwas bewirken wollen, oder wie Jäger (1986: 10) sagt:

Unmittelbares Ziel eines jeden Kommunikationsprozesses ist, beim Adressaten eine bestimmte Veränderung seines Bewußtseinszustandes zu bewirken (was seinerseits den Zweck haben kann, das Ziel einer übergeordneten Handlung bzw. Tätigkeit zu erreichen) (Jäger 1986: 10).

Somit liegt der Schwerpunkt des Übersetzens nicht im wörtlich Gesagten, sondern das Augenmerk des Übersetzens muss auf eine sprachlich repräsentierte Mitteilungsabsicht als mental-kognitive Größe gerichtet sein. Aus der Sicht des Autoren spricht man hinsichtlich der repräsentativen Funktion eines Textes von der Aussageintention und aus der Sicht des Empfängers in Bezug auf die kommunikative Funktion vom Textsinn.

\subsection{Der kommunikative Wert als Ausgangspunkt des Übersetzens}

Wie wir gesehen haben, wird eine Sprache dazu benutzt, etwas beim Hörer auszulösen, etwas zu bewirken, ihn über etwas zu informieren, ihn zu warnen, ihn zu loben, etc., was allgemein als die illokutive Funtion des Textes angesehen wird (Jung 2011: 128ff.). Somit ist der eigentliche Bezugspunkt bei der Übersetzung nicht der AT an sich, sondern dessen beabsichtigte Wirkung, die sich in einem Text durch die benutzten Wörtern manifestiert, indem der Empfänger diese interpretiert. Denn beim Übermitteln einer Aussageabsicht und dem Verstehen einer Aussage stützen sich Verfasser und Empfänger auf eine kognitive Größe des Ausgesagten, auf den kommunikativen Wert (vgl. De la Cruz 2013), der nach Jäger und Müller (1982: 44) zu verstehen ist als

die Eigenschaft eines Textes, mit einem bestimmten Abbildkomplex im Bewusstsein verknüpft bzw. verknüpfbar zu sein, d.h. die Eigenschaft, kraft der ein Text in der Kommunikation für einen Abbildkomplex "gesetzt" werden kann bzw. einen bestimmten Abbildkomplex auszulösen vermag (Jäger \& Müller 1982: 44).

Jeder Text hat also eine repräsentativ-kommunikative Funktion, es kommt ihm ein kommunikativer Wert zu und seine Textworte bilden das "sprachliche Substrat des kommunikativen Wertes" (Jäger 1986: 13). Damit ist gleichzeitig mit ausgesagt, dass der eigentliche Bezugspunkt bei der Übersetzung nicht der AT an sich ist, nicht die Wörter und ihre Bedeutungen bilden die Grundlagen des Übersetzens, sondern der durch den AT repräsentierte kommunikative Wert, den der Übersetzer durch einen ZT auf 
eine neue Art und Weise wiedergibt und zwar so, dass die Kommunikation gemäß der Intention des AT-Autoren mit einem zielsprachigen Empfänger zustande kommen kann (Neubert 1977: 15).

Ein Übersetzungsbeispiel möge kurz verdeutlichen, um was es beim kommunikativen Wert geht. In der spanischen und deutschen Kultur besteht selbstverständlich eine Woche aus sieben Tagen. Wenn nun aber ein Sprecher sich auf einen Zeitraum von zwei Wochen bezieht, so sagt er im Spanischen hoy en quince dias (heute in fünfzehn Tagen), und wenn er sich auf eine Woche bezieht, dann verwendet er den Ausdruck hoy en siete dias (heute in sieben Tagen). Soll man nun dies ins Deutsche übersetzen, so muss man beachten, dass das Deutsche in beiden Fällen genau umgekehrt zählt wie das Spanische, denn im Deutschen wird im bezug auf eine Woche der heutige Tag mitgerechnet, aber hinsichtlich von zwei Wochen nicht, und daher sagt man im Deutschen heute in vierzehn Tagen und heute in acht Tagen. Auch wenn sich die numerischen Bezeichnungen im Spanischen und Deutschen in diesen beiden Fällen nicht entsprechen, ist die mental-kognitive Göße, der kommunikative Wert dergleiche. Nicht das mathematisch Exakte interessiert als Bezugspunkt der Entsprechung beim Übersetzen, sondern das, was gemeint ist, eben der Zeitraum, der in beiden Kulturen unterschiedlichen Zählweisen unterliegt. Nur so bleibt der kommunikative Wert und dessen Wirkung im Spanischen und Deutschen erhalten. Alles andere führte zu Verwirrungen. Diese Art von Gleichwertigkeit ist gemeint, wenn man von der kommunikativen Äquivalenz spricht, d.h. im Hinblick auf die Kommunikation und auf nichts anderes versucht der Übersetzer einen ZT zu erstellen, der wie der AT funktioniert.

\subsection{Die kommunikative Äquivalenz als Ziel der Übersetzung}

Es ist deutlich geworden, dass beim Übersetzen nicht die Wörter eines Textes im Vordergrund stehen, sondern die Aussageabsicht oder eben der kommunikative Wert, der mittels der Textinterpetation vom Adressaten ermittelt wird. Dasselbe gilt dann vom Übersetzer, dessen Aufgabe nun darin besteht, die Kommunikation zwischen zwei Gesprächspartnern, die über keine gemeinsame Sprache verfügen, zu sichern, indem er einen kommunikativ gleichwertigen Text in der Zielsprache erstellt, dessen kommunikativen Eigenschaften die kommunikativen Eigenschaften des AT wiedergeben. Es ist Jäger (1986: 7) recht zu geben, wenn er das Übersetzen versteht als

eine komplexe sprachliche Tätigkeit im Rahmen der sprachlichen Kommunikation (...), die zu dem Zweck vollzogen wird, die Kommunikation zwischen ver- 
schiedensprachigen Partnern zu gewährleisten, und darin besteht, daß zu einem $\mathrm{L}_{\mathrm{Q}}$-Text (quellensprachigen Text) ein $\mathrm{Lz}_{\mathrm{z}}$-Text (zielsprachiger Text) geschaffen wird, der sich hinsichtlich seiner kommunikativ relevanten Eigenschaften auf die kommunikativ relevanten Eigenschaften des $\mathrm{L}_{\mathrm{Q}}$-Textes gründet, so daß der Lz-Text den Kommunikationsbedürfnissen von Adressat(en) und/oder $\mathrm{L}_{\mathrm{Q}}$-TextVerfasser bezüglich kommunikativ relevanter Eigenschaften des $\mathrm{L}_{\mathrm{Q}}$-Textes in dem Maße entspricht, wie dies unter den jeweiligen Bedingungen, unter denen die Sprachmittlung stattfindet, möglich ist (Jäger 1986: 7).

Das Übersetzen lässt sich demnach nicht auf einen rein linguistischen Beschreibungsansatz der Translation reduzieren, wie es oft der Leipziger Schule vorgeworfen wird (Snell-Hornby 1988: 20; Stolze 1994: 44f.), sondern Jäger versteht das Übersetzen als eine kognitiv basierte Tätigkeit, hebt mit Nachdruck die kommunikationsrelevanten Eigenschaften des AT im Hinblick auf den ZT hervor und nennt auch die jeweiligen Bedingungen beim Zustandekommen einer Übersetzung als entscheidende Faktoren, wobei er das Wesentliche der Translation im nicht-sprachlichen Bereich sieht. Im Zusammenhang mit dem intersemiotischen Übersetzen ist jetzt schon zu unterstreichen, dass die Bedürfnisse des ZT-Adressaten in den Vordergrund rücken und eben nicht der AT an sich.

Im Hinblick auf den ZT-Adressaten stellt sich nun die Frage, wie nun der Übersetzer es erreichen kann, dass der ZT dem AT kommunikativ äquivalent wird? Denn wie wir gesehen haben, kann er nicht einmal einfach die Zahlen direkt in die andere Sprache übernehmen, ohne zu überprüfen, ob der kommunikative Effekt der gleiche wäre. Worauf der Übersetzer zu achten hat, oder was die kommunikative Äquivalenz ausmacht, verdeutlicht die Defintion von Jäger (1975: 37):

Als kommunikativ äquivalent betrachten wir zwei Texte verschiedener Sprachen dann, wenn ein ideal zweisprachiger Sprecher (...) in der Kommunikation mit einem ebenso idealen Adressaten (...) die freie Wahl hat, den Text der Sprache LA oder den Text der Sprache LB zur Realisierung seiner Intention zur Äußerung zu verwenden, da beide Texte beim Adressaten denselben kommunikativen Effekt auslösen (Jäger 1975: 37).

Auf der Suche nach einem dem AT kommunikativ äquivalenten ZT muss sich also der Übersetzer darüber im Klaren sein, welche Vorkenntnisse er bei dem ZT-Adressaten voraussetzen kann und welches Wissen er ergänzen muss, damit dieser annähernd die gleichen Möglichkeiten der Textinterpretation (Siever 2010; 2015: 124-132) und damit des Textverstehens hat wie der Adressat des AT. Nur wenn der kommunikative Wert des AT und des ZT annähernd übereinstimmen, können wir überhaupt von einer gelungenen Übersetzung sprechen, denn dann besteht zwischen beiden Texten 
eine Beziehung, die sich im Sinne der Leipziger Übersetzungswissenschaftlichen Schule mit der kommunikativen Äquivalenz beschreiben lässt (Wotjak 2012).

Welche Kenntisse und welches Wissen von einem Adressaten eingebracht werden müssen, um einen Text überhaupt im Sinne des Autoren interpretieren zu können, und die folglich bei der Übersetzung zu beachten sind, lassen sich aus der nachfolgenden, aber sicher nicht vollständigen Liste von mindestens sechs Punkten ersehen:

1. Allgemeine Kenntisse der Sprache, die einen korrekten Sprachgebrauch erlauben (z.B. innerhalb der Grammatik die Bildung der unregelmäßigen Verben oder innerhalb der Syntax die deutsche Satzklammer).

2. Allgemeines Weltwissen (z.B. die Erde dreht sich um die Sonne).

3. Kulturwissen, das für die Kommunikation relevant ist (z.B. sogenannte Realien als verinnerlichtes Wissen einer Kultur, das sich in Symbolen, Sitten und Gebräuchen manifestiert, oder das Wissen, wie eine Kulturgemeinschaft das behandelte Thema bewertet).

4. Die allgemeine Sachkenntnis im Hinblick auf das behandelte Thema.

5. Das persönliche Wissen, das sich auf die Lebenswelt des Adressaten und / oder des Autoren bezieht.

6. Die Textkompetenz des Adressaten im Diskurs (z.B. Kohärenz und Kohäsion).

Demzufolge arbeitet ein Übersetzer innerhalb eines vorgesteckten Rahmens, nämlich der neuen Kommunikationssituation des ZT, die dadurch gekennzeichnet ist, dass die Kommunikation im Sinne des AT-Autoren bezüglich des ZT-Empfängers gewährleistet sein soll. Unterschiedliche Verstehensvoraussetzungen bezüglich des kulturellen, geschichtlichen und soziologischen Hintergrunds müssen im ZT derart berücksichtigt werden, dass der kommunikative Wert des ZT dem des AT entspricht und gleichzeitig ein ZT entsteht, der sprachlich korrekt und ohne Verstöße z.B. gegen Textkonventionen der Zielsprache (ZS) verfasst ist (Neubert 1977: 22).

In unserem Zusammenhang ist es nun besonders wichtig zu sehen, die kommunikative Äquivalenz setzt voraus, dass AT und ZT über nahezu den gleichen kommunikativen Wert verfügen, d.h. die gleichen Interpretationsmöglichkeiten aufweisen (Siever 2015: 128), die sich auch auf die vorher genannten Wissensliste stützen. Da jedoch oft das Allgemeinwissen, das man beim zielsprachigen Empfänger im Vergleich zum ausgangssprachlichen voraussetzen kann, aus historischen, kulturellen und soziologischen Unterschieden divergiert, bedeutet dies, dass wir bei der kommunikativen Äquivalenz von einem fast nie auftretenden Idealfall des Übersetzens sprechen, der aber als theoretisches Konstrukt unablässig ist, um beurteilen zu können, was unter Übersetzen zu verstehen ist. Der kommunikative Wert 
des ZT nähert sich immer nur an den kommunikativen Wert des AT an, ohne ihn je zu erreichen. Dennoch gilt es aber auch zu bedenken, dass der intendierte kommunikative Effekt des Autoren und der vom Empfänger aktualisierte kommunikative Wert ",auch in der einsprachigen Kommunikation kaum je völlig kongruieren, wohl aber einen die soziale Interaktion, die Verständigung - im vorgegebenen Situationskontext - gewährleistenden gemeinsamen Durchschnitt aufweisen“ (Jäger 1990: 273).

\section{ZUM INTERSEMIOTISCHEN ÜBERSETZEN DER AUDIODESKRIPTION}

In unseren Ausführungen konnte aufgezeigt werden, dass die Grundlagen des interlinguistischen Übersetzens nicht im rein Sprachlichen liegen, sondern vor allem im kognitiven Bereich. Die durch die Sprache geschaffenen und evozierten Bewusstseinsinhalte sind Ausgang und Ziel des Übersetzungsvorgangs. Im Folgenden soll nun gezeigt werden, wie sich diese Erkenntnisse auf die Prozesse des intersemiotischen Übersetzens der Audiodeskription (AD) zumindest teilweise übertragen lassen.

Die natürliche Sprache ist erfahrungsgemäß das allumfassende universelle Kommunikationsmittel, dessen Zeichen für jeden beliebigen menschlichen Bewusstseinsinhalt stehen kann, und ihre Universalität zeigt sich in der Fähigkeit, die vielfältigen und nahezu unbegrenzten Bereiche der menschlichen Kommunikationsbedürfnisse, also somit auch rein nicht-sprachliche Kommunikationsweisen wie z.B. in der Bildenden Kunst, der Musik oder dem Tanz, sprachlich zu erfassen. Die menschliche Sprache stellt demnach das bedeutendste und allgemeinste, jedoch nicht das einzige Instrument dar, mit dem wir unser Wirklichkeitswissen, das wir durch unsere Sinne über unsere Außenwelt erlangt haben, darstellen bzw. kodifizieren. Dieser Umstand ist die Grundlage dafür, jemandem durch ein sogenanntes intersemiotisches Übersetzen z.B. etwas Gehörtes oder Gesehenes mittels einer sprachlichen Kodifizierung zu kommunizieren. Wir können also z.B. durch Sprache ein Bild beschreiben (vgl. Soler 2012; Álvarez \& Jiménez 2016; Chica 2016) oder Musik im Einzelnen mit Worten wiedergeben (Martínez 2016), so dass der Hörer oder Leser sich selbständig eine angemessene Vorstellung des beschriebenen Bewusstseinsinhalts machen kann, also das Bild oder die Musik verständlich werden. Anhand der AD für Sehbehinderte möchten wir das intersemiotische Übersetzen, wie es in Filmen vorkommt, näher erläutern.

Es stellt sich somit die Frage, worauf denn der Audiobeschreiber bei seiner Bildwiedergabe zurückgreift, oder was den Textbausteinen der Über- 
setzung bei der $\mathrm{AD}$ entsprechen könnte. Aus Forschungsergebnissen verschiedener neurologischer Untersuchungen wissen wir, dass visuelle Wahrnehmung und Sprache im menschlichen Gehirn mit einander in enger Verbindung stehen. Mehrere Neurologen haben diesen Zusammenhang ausführlich beschrieben, der in gemeinsamen Verknüpfungen und Übertragungen zwischen einzelnen Bereichen des menschlichen Gehirns, die für das Sehen und das Sprechen zuständig sind, besteht (Martin, Haxby, Lalonde, Wiggs \& Ungerleider 1995; Liebermann 2002; Chica 2016: 41-79). Die visuelle Wahrnehmung und die menschliche Sprache sind beide ein Resultat neurologischer Hirnaktivitäten, die untereinander verkoppelt sind. Diese Erkenntnisse macht sich die $\mathrm{AD}$ zunutze und stützt sich folglich auf eine neurologisch grundgelegte Übersetzungspraxis, welche die konzeptuellen und semantischen Strukturen, die den Verstehensprozessen im menschlichen Gehirn gemeinsam sind, dazu verwendet, das durch die visuelle Wahrnehmung erworbene Wissen sprachlich zu kodifizieren. Grundlage dieser audiodeskriptiven Versprachlichung ist die Beschreibung von visuellen Elementen, die in unserem Beispiel für das Erfassen der Wirklichkeit innerhalb eines Filmereignisses kommunikativ relevant sind. Somit beruht die Wissensrepräsentation in einer vorgetragenen $\mathrm{AD}$ auf der außergewöhnlichen Fähigkeit des menschlichen Gehirns, visuelle Elemente durch die Kortexareale zu be- und zu verarbeiten und sie dann in Sekundenschnelle nahezu gleichzeitig auf höher gelegenen Ebenen anzuheben, wo in einem spezifischeren Verarbeitungsprozesses diese visuellen Elemente nach konzeptuellen und semantischen Kategorien geordnet und verknüpft werden, so dass visuell erworbenes Wissen mit sprachlichen Kodifizierungen in Verbindung gebracht werden können (Chica 2016: 133-139). In einer analogen Art und Weise bedeutet dies für unseren Zusammenhang, wie der ZT bei einer interlinguistischen Übersetzung in seinen kommunikativen Eigenschaften mit dem AT übereinstimmen soll, so hat die $\mathrm{AD}$ ein annähernd kommunikativ äquivalentes Bild bei einer sehbehinderten Person hervorzurufen, das der Filmvorlage entspricht. Um dies besser erklären zu können, müssen wir uns erst kurz deutlich machen, wie das menschliche Gehirn ein Bild überhaupt wahrnimmt.

\subsection{Mikroelemente der Bilderfassung}

Die Bildende Kunst versteht Farbe, Licht und Form als konstituive Komponenten visueller Wahrnehmung, die ineinander übergehen und erst in ihrem Zusammenspiel ein Bild entstehen lassen, was sich aus neurowissenschaftlichen Forschungen bestätigen lässt. Im menschlichen Gehirn gibt es 
nämlich unterschiedliche spezialisierte Bereiche, die unabhängig voneinander tätig sind und den Ausgangspunkt dafür bilden, auf visuelles Wissen, das auf unterschiedliche Hirnareale verteilt ist, zugreifen zu können (Zeki 2001). Einige Bildaspekte werden dabei vor anderen perzipiert, d.h., einzelne visuelle Komponenten werden zeitlich nacheinander wahrgenommen, z.B. zuerst erfasst das Gehirn Farbe und Form, und dann erst Bewegung (Moutoussis \& Zeki 1997). In verschiedenen neurologischen Studien konnte festgestellt werden, dass die strukturellen Mikroelemente der Bilderfassung eben Farbe, Form, Bewegung, Sehtiefe und Textur sind, und diese sind gerade für die $\mathrm{AD}$ die wichtigsten Elemente, die sprachlich adäquat in einer Bildbeschreibung dargestellt werden müssen. Erst mit diesen Grundinformationen kann unser Gehirn dazu übergehen, ein Bild zusammenzustellen bzw. diese visuellen Daten zu interpretieren, so dass wir von visuellen Makroelementen wie Objekt und Kontext sprechen können (Chica 2016: 141-158).

Übertragen auf die intersemiotische Übersetzung bedeutet dies für uns, dass der intendierte kommunikative Effekt bzw. der kommunikative Wert der AD sich aus der sprachlichen Darstellung dieser visuellen Komponenten, so wie sie das Gerhirn verarbeitet, zusammensetzt. Farbe, Form, Bewegung, Sehtiefe und Textur gelten als Grundbausteine für ein mentales Bild und als solches können sie dann auch sprachlich benannt und untereinander in Beziehung gesetzt werden. Hat die AD das Bild korrekt nach seinen visuellen Mikroelementen beschrieben, dann kann man davon ausgehen, dass eine sehbehinderte Person sich ein kommunikativ äquivalentes Bild einer Filmszene machen kann, indem sie diese Bildinformationen kohärent auf den zentralen visuellen Punkt hin interpretiert, nämlich das visuelle Objekt in seinem Kontext.

\subsection{Makroelemente der Bilderfassung: visuelles Objekt und Kontext}

Nach Erfassung der sogenannten Mikroelementen der Bildkomposition werden diese Informationen im mentalen Verarbeitungsprozess auf eine höhere Ebene gebracht, geordnet und wieder zusammengefügt, indem sie zueinander in Beziehung gesetzt werden. So entstehen in einem Bildkontext die visuellen Objekte. Man könnte auch sagen, das Gehirn interpretiert die Informationsdaten und bündelt die Bildelemente zu einem Kontext und zu einem visuellen Objekt (Chica 2016: 149ff.).

Das visuelle Objekt beruht auf der interpretativen Komposition der strukturellen Basiselementen und lässt sich anhand von konkreten und unterscheidbaren Merkmalen erkennen. Dieses Objekt kann als Aktant eine 
aktive oder passive Rolle in einem visuellen Geschehen einnehmen. Anders ausgedrückt, ein visuelles Objekt ist der Interpretationspunkt in einem Bild, um den sich die Mikroelemente wie Farbe, Form, Bewegung, Sehtiefe und Textur anordnen. Das menschliche Gehirn kombiniert die einzelnen Bildelemente, deutet die Zusammenhänge und bestimmt, was den zentralen Punkt in einem bildhaften Bewusstseinsinhalt ausmacht. Diese Zusammenhänge und Beziehungen unter den einzelnen Bildelementen lassen sich kategorisieren und werden dann in die Sprache übernommen, was sich dann allgemein in der Grammatik als semantische und syntaktische Funktionen, wie z.B. Aktant, Subjekt, Objekt, Thema, Rhema; etc., umschreiben lässt. Gleichzeitig ist das visuelle Objekt in ein übergeordnetes Wahrnehmungsereignis eingebettet und grenzt sich davon als Bezugspunkt ab, d.h. die Kategorie Objekt definiert eine zweite Kategorie, den Kontext, und beide sind von einander abhängig und bedingen sich gegenseitig (Bar 2004).

Führen wir ein kurzes Beispiel an, um diese zwei Makroelemente der visuellen Wahrnehmung zu verdeutlichen. Angenommen wir sehen in der Autowerbung ein Bild, wo im Vordergrund ein roter Geländewagen gezeigt wird und im Hintegrund befindet sich ein See in den Alpen. In diesem Zusammenhang wäre klar, dass der zentrale Bezugspunkt des Bildes der Geländewagen als visuelles Objekt ist, denn das Gehirn verarbeitet die vorgegebene Information Autowerbung und konzentriert sich auf den Geländewagen, den das Gehirn mit seinen Merkmalen wie Farbe, Form, etc. wahrnimmt und zwar im Kontrast zum Hintergrund bzw. dem visuellen Kontext, dem Alpensee.

Das gleiche Bild würde nun von unserem Gehirn anders interpretiert werden, wenn wir uns einen Dokumentarfilm anschauten, denn dann wäre der Geländewagen eher ein sekundäres visuelles Objekt bzw. gehörte zum Kontext, wohingegen der Alpensee das eigentliche visuelle Objekt wäre. Wie beim Übersetzen beeinflussen also die Vorkenntnisse das Interpretationsverhalten des Adressaten und somit den kommunikativen Wert oder visuellen Wert, der im menschlichen Gehirn verarbeitet wird.

Wir können also festhalten, die Kategorie visuelles Objekt ist eine der strukturellen Grundelemente für die Wahrnehmung und das Verstehen eines komplexen visuellen Geschehens. Das Objekt und seine Merkmale bestimmen den Kontext. Visuelles Objekt und visueller Kontext grenzen sich innerhalb der ganzen Szenerie ab, wobei dann beide Kategorien das Fundament für eine intersemiotische Übertragung des visuellen Inhalts auf die Sprachebene innerhalb des Gehirns bilden (Chica Núñez 2016: 158-161).

Visuelles Objekt und Kontext fundieren auf der Basis der visuellen Grundbausteine (besonders Farbe, Form und Bewegung) als entscheidende Faktoren eines Bildereignisses. Die visuellen Grundbausteine werden so 
beschrieben, dass das visuelle Objekt als der Kern eines kognitiven Schemas erkennbar wird, wie dies aus der scence-and-frame-semantics von Fillmore (1977) oder dem Handlungsmusterwissen von Wotjak $(1987 ; 1988)$ in der Übersetzungswissenschaft bekannt ist (Jiménez \& Seibel 2004). Demnach kann schon allein durch einen Fachausdruck eine vollständige Repräsentation eines Geschehens beim Empfänger evoziert werden. Mit anderen Worten, die AD darf sich nicht in detailverliebten Bildbeschreibungen verlieren, sondern sollte sich dessen bewusst, dass die Sprache immer eine kommunikative Funktion ausübt und entsprechend den Erkennungsmechanismen des Gehirns Informationen an die sehbehinderte Person weitergibt. Das bedeutet, die AD schafft einen visuellen Rahmen, um ein bestimmtes Szenario als kommunikativen Wert durch Sprache in einem ZT als Textwelt erstehen zu lassen. Der visuelle Rahmen umfasst dabei einzelne Szenarien, die sich wiederum aus unterschiedlichen visuellen Objekten (eigenständigen Elementen mit bestimmten Aktionspotential wie z.B. ein Motorrad, ein Affe oder ein Schiedsrichter in einem weiter gefassten visuellen Kontext (z.B. im Straßenverkehr, in einem Forschungsinstitut oder auf einem Fussballplatz) zusammensetzen, und auf diese Art und Weise verstehbar werden, da sie als Handlungsmuster oder kognitives Schema vom menschlichen Hirn abgerufen werden können.

\section{SCHLUSS}

Ziel dieses Artikels war es, Verbindungen zwischen dem interlinguistischen und intersemiotischen Übersetzen aufzuzeigen. Um zu erklären, was Übersetzen eigentlich ist, wurde aufgezeigt, wie Sprache funktioniert, nämlich indem sie sich auf die beiden zu leistenden Grundaufgaben der repräsentativen und kommunikativen Funktion stützt. Für das interlinguistische Übersetzen bedeutet dies, dass der Übersetzer einen ZT erstellt, dessen kommunikativen Eigenschaften denen des AT entspricht, so dass ZT und AT zueinander in einer Beziehung stehen, die sich mit dem Begriff der kommunikativen Äquivalenz beschreiben lässt (vgl. Wotjak 2012).

In einem zweiten Schritt wurde aufgezeigt, wie das menschliche Gehirn visuelle Elemente wahrnimmt und verarbeitet. Wichtig war dabei festzustellen, dass es Verbindungen zwischen der Verarbeitung visueller Elemente und den sprachlichen Konzepten gibt, was uns ermöglicht, den Informationsprozess auf der sprachlichen Ebene als eine Repräsentation semantischvisueller Information(-en) aufzufassen, was als Darstellung von Wissen verstanden wird. Übertragen auf die AD können wir sagen, die audiodeskriptive Textproduktion beruht auf der Versprachlichung von Beziehungen, die 
unter visuellen Elementen bei ihrer Wahrnehmung im menschlichen Gehirn entstehen. Dabei impliziert diese Darstellungsweise eine Transformation des Repräsentationsmodus, denn das durch Bilder visuell erworbene Wissen wird auf eine natürliche Sprache übertragen. Demnach ist die AD ein Übersetzungsprozess, bei dem das erworbene Wissen über visuell wahrgenommene Elemente (Farbe, Bewegung, Form, Objekt) sprachlich zum Ausdruck kommt. Wissen wird dargestellt, indem durch Sprache Beschreibungen der Wirklichkeit vorgenommen werden, die eine gewisse Übereinstimmung mit einem Realitätszustand aufweisen (Brachman \& Lavesque 1985). Im Ablauf einer $\mathrm{AD}$ besteht somit die Wissensrepräsentation darin, visuelle Informationen schnell mittels der kognitiven Strukturen, über die das Gehirn verfügt, $\mathrm{zu}$ assimilieren und diese Informationen auf spezifischeren Hirnebenen weiter zu verarbeiten, wobei gleichzeitig von der Fähigkeit des Hirns auszugehen ist, dieses Wissen mit bestimmten sprachlichen Repräsentationen zu verbinden. Dieser Verarbeitungsprozess von Wissen impliziert die Abspeicherung von visuell wahrgenommenen Elementen durch Begriffe im Gedächtnis und deren Assimilation im mentalen Lexikon, ein Automatismus, der aus dem Umgang mit fachsprachlichen Ausdrücken, der Kreativität bei der Formulierung neuer Termini und der sprachlichen Ausdrucksweise bekannt ist (vgl. Jung 2010; Faber \& León-Araúz 2016) und es folglich rechtfertigt, die AD als eine Art des intersemiotischen Übersetzens zu verstehen. Denn wie der Übersetzer von Wörtern, Sätzen und einen Gesamttext ausgeht, um den Textsinn zu erschließen, so setzt unser Gehirn die Bildelemente Farbe, Form, Bewegung, Tiefe und Textur wie Bausteine zu einem Bild zusammen. Diese Bildinformationen lassen sich als grammatikalische Beziehungen deuten und für die audiodeskriptive Textproduktion nutzen.

\section{LITERATURVERZEICHNIS}

Aguiar, D. / Queiroz, J. (2013). Semiosis and intersemiotic translation. Semiotica, 196, 283-292.

Aktulum, K. (2017). What is intersemiotics? A short definition and some examples. International Journal of Social Science and Humanity, 7 (1), 33-36. DOI: 10.18178/ijssh.2017.7.1.791 (abgerufen am 15.02.2018).

Álvarez, C. / Jiménez, C. (Hrsg.) (2016). Patrimonio cultural para todos. Investigación aplicada en traducción accesible. Granada: Tragacanto.

Bar, M. (2004). Visual objects in context. Nature Reviews Neuroscience, 5 (8), 617-629.

Brachman, R. J. / Levesque, H. J. (1985). Readings in knowledge representation. Los Altos: Kaufmann

Chica, A. J. (2016). La traducción de la imagen dinámica en textos multimodales. Granada: Tragacanto.

Da Silva, A.C. (2017). On Jakobson's intersemiotic translations in Asterix comics. Comparatismi, 2, 71-81. DOI: dx.doi.org/10.14672/20171233 (abgerufen am 15.03.2018). 
De la Cruz, M. (2013). El concepto de valor comunicativo en la Escuela Traductológica de Leipzig: Gert Jäger. Una propuesta teórica de ampliación pragmático-hermenéutica. Granada: Universidad de Granada. http:/ / digibug.ugr.es/handle/10481/29543 (abgerufen am 20.11.2017).

Faber, P. / León-Araúz, P. (2016). Specialized knowledge representation and the parameterizetion of context. Frontiers in Psychology, 7 (00196). DOI:10.3389/fpsyg.2016.00196 (abgerufen am 20.07.2017).

Fernández, E. / Martínez, S. / Chica, A. J. (2015). Cross-fertilisation between reception studies in audio description and interpreting quality assessment: The role of the describer's voice. In: R. Baños Piñero / J. Díaz Cintas (Hrsg.), Audiovisual Translation in a Global Context (S. 72-95). London: Palgrave Macmillan.

Fillmore, C. (1977). Scences-and-frames-semantics. In: A. Zampolli (Hrsg.), Linguistic Structures Processing (S. 55-81). Amsterdam: North-Holland.

Jäger, G. (1975). Translation und Translationslinguistik. Halle (Saale): Niemeyer.

Jäger, G. (1986). Die sprachliche Bedeutung - das zentrale Problem bei der Translation und ihrer wissenschaftlichen Beschreibung. In: G. Jäger / A. Neubert (Hrsg.), Bedeutung und Translation (S. 5-66). Leipzig: Verl. Enzyklopädie.

Jäger, G. (1990). Überlegungen zur kommunikativen Äquivalenz. In: H. Salevsky (Hrsg.), Übersetzungswissenschaft und Sprachmittlung. Band II (S. 272-277). Berlin: Humboldt-Universität.

Jakobson, R. (1959). On linguistics aspects of translation. In: R. Brower (Hrsg.), On translation (S. 232-239). Cambridge (Mass.): Harvard University Press.

Jiménez, C. / Martínez Martínez, S. (2018). Leisure and culture accessibility: the OPERA Project. Cultus Journal (im Druck).

Jiménez, C. / Seibel, C. (2004). Modelos culturales activados en los textos especializados: hacia un concepto de representación del conocimiento experto. In: E. Fleischmann / P. Schmitt / G. Wotjak (Hrsg.), Translationskompetenz (S. 511-523). Tübingen: Stauffenburg.

Jung, L. (2010). Fachsprache Deutsch: die Darstellung von Begriffsbeziehungen in Fachtexten. LSP Journal, 1 (1), 47-60.

Jung, L. (2011). Die Kulturkompetenz im Übersetzungsunterricht: vom Wort zum Text. In: S. Adamczak-Krysztofowicz et al. (Hrsg.), Aktuelle Probleme der angewandten Linguistik. Interkulturalität als Schlüsselkompetenz für Fremdsprachenlehrer, Übersetzer und Mediatoren (S. 123-136). Poznań: Wydawnictwo Naukowe UAM.

Kade, O. (1963). Aufgaben der Übersetzungswissenschaft. Fremdsprachen, 2, 83-94.

Kopetzki, A. (2015). Praxis und Theorie des literarischen Übersetzens: Neue Perspektiven. In: A. Buschmann (Hrsg.), Gutes Übersetzen. Neue Perspektiven für Theorie und Praxis des Literaturübersetzens (S. 69-84). Berlin: De Gruyter.

Kvam, S. (2014). Zur Übersetzung von intersemiotischen Texten am Beispiel von Kunstliedern. Eine pragmatisch-textlinguistische Analyse. Trans-kom, 7 (1), 115-139. http://www.transkom.eu/bd07nr01/trans-kom_07_01_06_Kvam_Kunstlied.20140606.pdf (abgerufen am 25.06.2017).

Lieberman, P. (2002). On the nature and evolution of the neural bases of human language. American Journal of Physical Anthropology, 119 (35), 36-62.

Limbach, C. (2012). La neutralidad en la audiodescripción filmica desde un punto de vista traductológico. Granada: Universidad de Granada. http://digibug.ugr.es/handle/10481/24487 (abgerufen am 22.09.2017).

Martin, A. / Haxby, J. / Lalonde, F. / Wiggs, C. / Ungerleider, L. (1995). Discrete cortical regions associated with knowledge of color and knowledge of action. Science, 270, 102-105. 
Martínez, S. (2016). El sonido: el gran desconocido. El texto origen en el Subtitulado para Sordos. Revista Académica Liletrad, 1, 569-579.

Moutoussis, K. / Zeki, S. (1997). Functional segregation and temporal hierarchy of the visual perceptive systems. Procceedings of the Royal Society of London, B 264, 1407-1414.

Neubert, A. (1977). Zur kommunikativen Äquivalenz. Linguistische Arbeitsberichte, 16, 15-22.

Siever, H. (2010). Übersetzen und Interpretation: die Herausbildung der Übersetzungswissenschaft als eigenständige wissenschaftliche Disziplin im deutschen Sprachraum von 1960 bis 2000. Frankfurt: Peter Lang.

Siever, H. (2015). Übersetzungswissenschaft. Eine Einführung. Tübingen: Narr Francke Attempto.

Snell-Hornby, M. (1988). Translation Studies. An Integrated Approach. Amsterdam-Philadelphia: John Benjamins.

Soler, S. (2012). Traducción y accesibilidad en el museo del siglo XXI. Granada: Tragacanto.

Stolze, R. (1994). Übersetzungstheorien: Eine Einführung. Tübingen: Narr.

Wotjak, G. (1987). Semantische Mikrostrukturen von Verben und kognitive Handlungsmuster. In: R. Crespo / B. Dotson Smith / H. Schultink (Hrsg.), Aspects of Language, Studies in Honour of Mario Alinei. Vol. II: Theoretical and Applied Semantics (S. 537-554). Amsterdam: Rodopi.

Wotjak, G. (1988). Lexikalische Bedeutungsbeschreibung und Handlungsmusterwissen. In: Universität Jena (Hrsg.), 2. Jenaer Semantik-Syntax-Symposium (S. 154-174). Jena: Universität Jena.

Wotjak, G. (2012). Äquivalenz und kein Ende? Nochmals zur semantischen, kommunikativen und translatorisch-diskursiven Äquivalenz. In: L. Jung (Hrsg.), Übersetzen als interdisziplinäre Herausforderung. Ausgewählte Schriften von Gerd Wotjak (S. 23-50). Frankfurt: Peter Lang.

Wotjak, G. (Hrsg.) (2006). 50 Jahre Leipziger Übersetzungswissenschaftliche Schule: Eine Rückschau anhand von ausgewählten Schriften und Textpassagen. Frankfurt a.M.: Lang.

Wotjak, G. / Sinner, C. / Jung, L. / Batista, J. (Hrsg.) (2013). La Escuela traductológica de Leipzig: sus inicios, su credo y su florecer (1965-1985). Frankfurt: Peter Lang.

Zeki, S. 2001. Localization and globalization in conscious vision. Annual Review of Neuroscience, $24(1), 57-86$.

Received: 13.06.2018; revised: 2.08 .2018 
